\title{
L'infection à Chlamydia trachomatis et le lymphogranulome vénérien au Canada :
} 2003-2012

\author{
Rapport sommaire \\ Totten $S^{1, *}$, MacLean $R^{1}$, Payne $E^{1}$, Severini $A^{2}$ \\ ${ }^{1}$ Centre de la lutte contre les maladies transmissibles et les infections, Agence de la santé publique du Canada, Ottawa \\ (Ontario) \\ ${ }^{2}$ Laboratoire national de microbiologie, Agence de la santé publique du Canada, Winnipeg (Manitoba) \\ *Auteure-ressource : Stephanie.Totten@phac-aspc.gc.ca
}

\section{Résumé}

Contexte : L'infection à Chlamydia trachomatis continue d'être l'infection transmissible sexuellement la plus couramment déclarée au Canada. Le lymphogranulome vénérien, causé par certains sérotypes de Chlamydia trachomatis, est en voie de devenir établi dans certaines populations dans un certain nombre de pays occidentaux.

Objectif : Déterminer les tendances parmi les cas déclarés d'infection à Chlamydia trachomatis et de lymphogranulome vénérien au Canada, du $1^{\mathrm{er}}$ janvier 2003 au 31 décembre 2012.

Méthodologie : Les données sur les maladies à déclaration obligatoire sur l'infection à Chlamydia trachomatis ont été présentées à l'Agence de la santé publique du Canada par les unités épidémiologiques provinciales et territoriales et résumées à l'échelle nationale en fonction de l'âge et du sexe. Les tests de confirmation des cas soupçonnés de lymphogranulome vénérien et le sous-typage du sérotype ont été effectués par le Laboratoire national de microbiologie (LNM). Lorsque c'est possible, les autorités sanitaires provinciales et territoriales utilisent un formulaire standardisé de déclaration de cas pour recueillir des données épidémiologiques améliorées sur chaque cas et pour soumettre les données à l'Agence.

Résultats : De 2003 à 2012, le taux de cas déclarés d'infection à Chlamydia trachomatis a augmenté de 57,6 \%, passant de 189,6 à 298,7 cas par 100000 habitants. Le taux de cas déclarés d'infection à Chlamydia trachomatis chez les femmes (383,5 cas par 100000 habitants) était près de deux fois supérieures à celui des hommes (212,0 cas par 100000 habitants), bien que la plus forte augmentation relative du taux a été observée chez les hommes. Chez les hommes comme chez les femmes, les taux d'infection à Chlamydia trachomatis les plus élevés ont été observés chez les personnes âgées de 20 à 24 ans. De 2004 à 2012, 170 cas de lymphogranulome vénérien ont été déclarés à l'Agence par les autorités sanitaires provinciales (dont 104 cas confirmés et 66 cas probables). En 2012, on a reçu des déclarations de 12 cas confirmés et probables, comparativement à 38 cas positifs confirmés par le Laboratoire national de microbiologie.

Conclusion : Au Canada, comme dans de nombreux pays, les taux d'infection à Chlamydia trachomatis ont sensiblement augmenté au cours des dix dernières années, en partie en raison de l'amélioration du diagnostic au moyen de tests d'amplification des acides nucléiques (TAAN). Conformément aux tendances observées en Europe et dans d'autres pays, le lymphogranulome vénérien est émergent au Canada chez les hommes ayant des relations sexuelles avec d'autres hommes (HARSAH).

\section{Introduction}

\section{Infection à Chlamydia trachomatis}

L'infection à Chlamydia trachomatis est à déclaration obligatoire à l'échelle nationale depuis 1991. II s'agit d'une infection génitale causée par la bactérie Chlamydia trachomatis et elle est le plus souvent déclarée comme une infection transmissible sexuellement (ITS) au Canada. Les infections sont souvent asymptomatiques chez les hommes comme chez les femmes. En l'absence de dépistage, ces infections ne sont pas diagnostiquées, ce qui 
favorise la propagation de l'infection à Chlamydia trachomatis parmi les personnes sexuellement actives (1). L'atteinte inflammatoire pelvienne est une des complications les plus courantes associées à l'infection à Chlamydia trachomatis non traitée et récurrente chez les femmes, et elle peut être à l'origine de douleurs pelviennes chroniques, de grossesses ectopiques et de cas de stérilité. Chez les hommes, les complications sont plus rares, mais elles comprennent des cas d'orchi-épididymite et de stérilité. Chez les femmes enceintes, l'infection à Chlamydia trachomatis non traitée peut être transmise au bébé à l'accouchement et causer une conjonctivite ou une pneumonie néonatales. Comme c'est le cas pour les autres infections transmissibles sexuellement, l'infection à Chlamydia trachomatis accroît le taux d'infection et de transmission du virus de l'immunodéficience humaine $(\mathrm{VIH})$. Elle recrute des cellules cibles pour le VIH dans le tractus génital et fait augmenter l'excrétion de cellules infectées au $\mathrm{VIH}(2,3)$.

Entre 1991 et 1997, le taux de cas déclarés d'infection à Chlamydia trachomatis a constamment diminué chez les hommes et chez les femmes, pour ensuite recommencer à augmenter (4).

\section{Lymphogranulome vénérien}

Le lymphogranulome vénérien est une ITS causée par les sérotypes L1, L2 et L3 de C. trachomatis. Les infections causées par ces sérotypes envahissent de préférence les tissus lymphatiques et elles ont tendance à se propager davantage que celles causées par les souches de Chlamydia trachomatis autres que celles du lymphogranulome vénérien. Les signes et les symptômes comprennent une rectite ulcéreuse douloureuse, accompagnée d'une lymphadénopathie inguinale ou fémorale ou des bubons, accompagnés de fièvre, de myalgie et d'arthralgie.

Le lymphogranulome vénérien non traité peut entraîner des complications graves, notamment la destruction des tissus rectaux et génitaux. Bien que rares, dans certains cas, une méningoencéphalite, une hépatite et la mort peuvent également survenir.

Le lymphogranulome vénérien est endémique dans certaines régions de l'Afrique, de l'Asie, de l'Amérique du Sud et des Caraïbes, et il était relativement rare au Canada jusqu'en 2003 (5). À cette époque, on a commencé à observer des éclosions de lymphogranulome vénérien chez les hommes ayant des relations sexuelles avec d'autres hommes dans des centres urbains canadiens (6). Des éclosions chez les HARSAH ont également été signalées dans des pays européens et aux États-Unis $(7,8,9,10)$. Des données récentes semblent indiquer que l'infection est devenue endémique dans la population des HARSAH dans certains de ces pays (11). En réponse à l'apparition du lymphogranulome vénérien en Europe, le Canada a lancé une surveillance accrue de cette ITS en 2005, qui incluait les données rétrospectives de 2004.

L'objectif du présent relevé est de résumer les tendances dans les taux d'infection à Chlamydia trachomatis et de lymphogranulome vénérien au Canada entre 2003 et 2012. II est fondé sur le rapport sur les infections transmissibles sexuellement au Canada : 2012, préparé par le Centre de la lutte contre les maladies transmissibles et les infections de l'Agence de la santé publique du Canada (ASPC) (accessible en ligne) (12) et une déclaration supplémentaire sur le lymphogranulome vénérien (13).

\section{Méthodologie}

\section{Collecte de données}

Les données sur les cas confirmés d'infection à Chlamydia trachomatis ont été déclarées au Système canadien de surveillance des maladies à déclaration obligatoire (SSMDO) par les autorités sanitaires provinciales et territoriales selon les définitions de cas de l'Agence pour les maladies transmissibles faisant l'objet d'une surveillance nationale (14) (voir l'encadré ci-dessous). Les données sont présentées sous des formes diverses (p. ex. transmission électronique de données présentées par ligne, exposés de cas sur support papier, données agrégées) et sont vérifiées et chargées dans la base de données du SSMDO par le personnel de l'Agence.

Les tests de confirmation des cas soupçonnés de lymphogranulome vénérien sont effectués par le Laboratoire national de microbiologie (LNM) au moyen du test d'amplification des acides nucléiques (TAAN), et des données sur ces cas sont ensuite partagées avec le Centre de la lutte contre les maladies transmissibles et les infections (CLCMTI). Le diagnostic des cas probables est fondé uniquement sur des tests sérologiques et il est effectué dans des laboratoires provinciaux. Lorsque c'est possible, les autorités sanitaires provinciales et territoriales 
utilisent un formulaire standardisé de déclaration de cas pour recueillir des données épidémiologiques améliorées sur les cas confirmés et probables, puis elles soumettent les données directement au CLCMTI. À I'heure actuelle, il n'est pas possible d'établir un lien entre les formulaires de déclaration de cas présentés au CLCMTI et les données correspondantes du LNM.

\section{Définitions de cas d'infection à Chlamydia trachomatis et de lymphogranulome vénérien}

\section{Infection à Chlamydia trachomatis : (14)}

Preuve de laboratoire d'infection des échantillons génito-urinaires et extra-génitaux :

- Détection de Chlamydia trachomatis par culture

OU

- Détection d'acides nucléiques de $C$. trachomatis

OU

- Détection d'antigène de $C$. trachomatis

Lymphogranulome vénérien: (13)

- Preuve de laboratoire des sérotypes L1, L2 et L3 de C. trachomatis par des TAAN.

\section{Analyse des données}

Les données sur l'infection à Chlamydia trachomatis extraites du SSMDO ont été analysées par le personnel du CLCMTI. Les taux ont été calculés selon le groupe d'âge et le sexe à l'aide des estimations de la population tirées de la Division de la démographie de Statistique Canada. Les calculs des taux de changements ont été effectués à partir de chiffres non arrondis pour une plus grande précision.

Le nombre de cas de lymphogranulome vénérien déclarés par le LNM et par les autorités sanitaires provinciales et territoriales est inclus séparément, étant donné qu'il n'existe actuellement aucune méthode permettant d'établir un lien entre ces deux sources de données. Les cas confirmés déclarés par le LNM peuvent créer des doublons de cas confirmés, déclarés également par les provinces, mais ce degré de chevauchement est difficile à quantifier.

La surveillance est considérée comme faisant partie du mandat des programmes fédéraux, provinciaux et territoriaux et ne s'inscrit pas dans le cadre de la recherche sur des humains; par conséquent, aucune approbation du Comité d'examen éthique n'a été demandée.

\section{Résultats}

\section{Infection à Chlamydia trachomatis}

En 2012, 103716 cas d'infection à Chlamydia trachomatis ont été signalés, ce qui correspond à un taux de 298,7 par 100000 habitants. Le taux en 2012 représentait une hausse de 57,6\% par rapport au taux de 189,6 par 100000 habitants en 2003. Chez les hommes, les taux ont augmenté de 74,8\%, passant de 121,3 à 212,0 cas par 100000 habitants; chez les femmes, les taux ont augmenté de 49,5\%, passant de 256,5 à 383,5 cas par 100000 habitants (Figure 1). 
Figure 1 : Taux de cas déclarés d'infection à Chlamydia trachomatis selon le sexe et dans l'ensemble, de 2003 à 2012, Canada

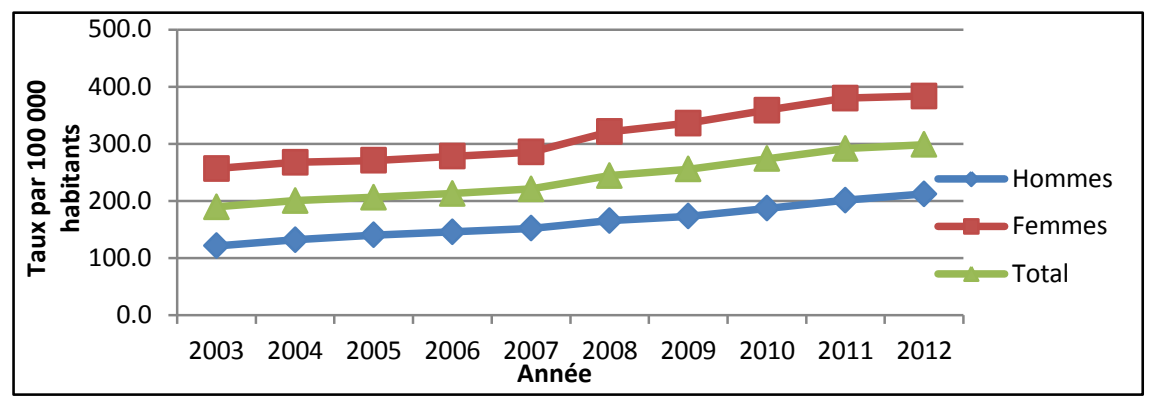

En 2012, les taux étaient presque deux fois plus élevés chez les femmes que chez les hommes et la majorité des infections à Chlamydia trachomatis déclarées (80,2 \%) touchaient des personnes de moins de 30 ans (Figure 2). En 2012, les taux les plus élevés étaient observés dans le groupe d'âge de 20 à 24 ans, bien que les taux chez les femmes étaient plus de deux fois plus élevés que les taux observés chez les hommes de ce groupe d'âge (2 151,7 cas par 100000 habitants c. 1073,9 cas par 100000 habitants, respectivement). Dans les groupes plus âgés, l'écart entre les sexes était moins prononcé et s'était même inversé; en 2012, les taux de cas déclarés étaient plus élevés chez les hommes que chez les femmes dans les groupes d'âge de 40 ans et plus (Figure 2).

Entre 2003 et 2012, les taux de cas déclarés d'infection à Chlamydia trachomatis n'ont cessé d'augmenter tant chez les femmes que chez les hommes âgés de 10 ans et plus. La plus forte augmentation relative des taux a été observée chez les personnes âgées de 10 à 14 ans (167,0\%), alors que la plus forte augmentation relative des taux chez les femmes a été observée chez celles âgées de 60 ans et plus (266,8 \%) (données non indiquées).

Figure 2 : Taux de cas déclarés d'infection à Chlamydia trachomatis selon le sexe et le groupe d'âge, 2012, Canada

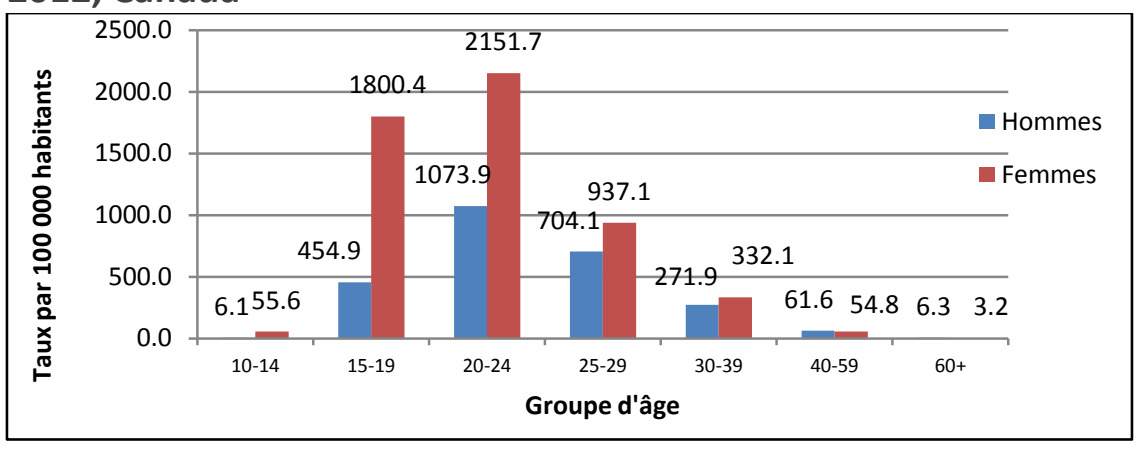

\section{Lymphogranulome vénérien}

En date de décembre 2012, les autorités sanitaires provinciales avaient déclaré 170 cas à l'Agence (dont 104 cas confirmés et 66 cas probables) (Tableau 1). Tous les cas confirmés ont été observés chez des hommes, principalement chez des HARSAH. Seulement en 2012, les provinces et les territoires ont déclaré huit cas confirmés et quatre cas probables. Toutefois, le LNM a déclaré 38 cas confirmés en laboratoire. 
Tableau 1 : Cas confirmés et probables déclarés de lymphogranulome vénérien, 2004-2012, Canada

\begin{tabular}{|c|c|c|c|}
\hline Année & $\begin{array}{l}\text { Confirmés } \\
\text { (LNM) }\end{array}$ & $\begin{array}{l}\text { Confirmés } \\
\text { (Formulaire de } \\
\text { déclaration de } \\
\text { cas) }\end{array}$ & $\begin{array}{l}\text { Probables } \\
\text { (Formulaire de } \\
\text { déclaration de } \\
\text { cas) }\end{array}$ \\
\hline 2004 & 1 & 3 & 7 \\
\hline 2005 & 37 & 36 & 21 \\
\hline 2006 & s. 0. & 26 & 16 \\
\hline 2007 & s. 0. & 1 & 7 \\
\hline 2008 & s. 0. & 1 & 4 \\
\hline 2009 & s. 0. & 9 & 0 \\
\hline 2010 & 18 & 9 & 2 \\
\hline 2011 & 34 & 11 & 5 \\
\hline 2012 & 38 & 8 & 4 \\
\hline Total & 128 & 104 & 66 \\
\hline
\end{tabular}

\section{Discussion}

On a observé une augmentation des taux de cas déclarés d'infection à Chlamydia trachomatis au Canada au cours de la dernière décennie. Plusieurs facteurs doivent être pris en compte dans l'analyse de ces données.

L'introduction de tests d'amplification des acides nucléiques (TAAN) plus sensibles au milieu des années 1990 a sans aucun doute entraîné une augmentation du nombre de cas détectés d'infection à Chlamydia trachomatis. Les tests d'amplification des acides nucléiques permettent d'utiliser des échantillons d'urine, qui sont plus faciles à recueillir; la méthode est également plus acceptable pour les patients que l'écouvillonnage. Par conséquent, le nombre de personnes qui se soumettent à un test a probablement augmenté aussi, surtout chez les hommes. Un dépistage plus efficace et une meilleure notification des partenaires ont peut-être eu un effet similaire $(15,16)$. Selon une récente estimation du fardeau de l'infection à Chlamydia trachomatis au Canada, l'augmentation de la prévalence de cette maladie pourrait s'expliquer par un dépistage efficace et par l'élargissement des programmes de dépistage (17).

Au fil du temps, le taux de cas déclarés d'infection à Chlamydia trachomatis a toujours été environ deux fois plus élevé chez les femmes que chez les hommes; cependant, cette disparité est beaucoup plus prononcée chez les groupes d'âge plus jeunes. Les jeunes femmes sont biologiquement plus vulnérables à l'infection à Chlamydia trachomatis $(18,19)$. En outre, les femmes sont plus susceptibles de passer un test de dépistage des infections transmissibles sexuellement $(20,21)$.

Les tendances en matière de lymphogranulome vénérien sont difficiles à interpréter en raison de lacunes dans les données disponibles, mais il a été démontré qu'il est en voie de devenir endémique chez les HARSAH au Canada. Les efforts de surveillance précoce ont été intensifs, suivis d'une période (2007-2009) au cours de laquelle peu de cas ont été déclarés. L'augmentation la plus récente des cas, qui a commencé en 2010 et s'est poursuivie en 2012, reflète probablement l'amélioration de la recherche et de la déclaration des cas (22).

Des lignes directrices nationales en matière de prévention et de prise en charge des cas d'infection à Chlamydia trachomatis sont disponibles $(2,23)$ et les lignes directrices relatives au traitement du lymphogranulome vénérien ont récemment été mises à jour (13).

\section{Remerciements}

Le relevé dans son intégralité a été préparé par le Centre de la lutte contre les maladies transmissibles et les infections de la Direction générale de la prévention et du contrôle des maladies infectieuses de l'Agence de la santé publique du Canada. Sa publication n'aurait pas été possible sans les données fournies par l'ensemble des provinces et territoires. Nous leur sommes très reconnaissants de leur contribution continue à la surveillance nationale des infections transmissibles sexuellement. Les auteurs sont également reconnaissants envers le Réseau de surveillance des infections transmissibles par le sang pour son expertise et sa contribution. 


\section{Conflit d'intérêts}

Aucun

\section{Financement}

Ce travail a été appuyé par l'Agence de la santé publique du Canada.

\section{Références}

(1) Stamm WE. Chlamydia trachomatis infections of the adult. In: Holmes KK, Sparling PF, Mardh P, Lemon SM, Stamm WE, Piot P, et al., editors. Sexually transmitted diseases. 3rd ed. New York: McGraw-Hill; 1999. p. 407-422.

(2) Expert Working Group for the Canadian Guidelines on Sexually Transmitted Infections. Chlamydial Infections. In: Wong T, Latham-Carmanico C, editors. Canadian guidelines on sexually transmitted infections. 2010 ed. Ottawa: Public Health Agency of Canada; 2010.

(3) Fleming DT, Wasserheit JN. From epidemiological synergy to public health policy and practice: the contribution of other sexually transmitted diseases to sexual transmission of HIV infection. Sex Transm Infect 1999 Feb; 75(1):3-17.

(4) Public Health Agency of Canada. Report on sexually transmitted infections in Canada: 2011. Centre for Communicable Diseases and Infection Control, Infectious Disease Prevention and Control Branch, Public Health Agency of Canada; 2014. http://publications.gc.ca/site/eng/469949/publication.html. (Disponible en français : http://publications.gc.ca/site/fra/469950/publication.html).

(5) Expert Working Group for the Canadian Guidelines on Sexually Transmitted Infections. Lymphogranuloma venereum (LGV). In: Wong T, Latham-Carmanico C, editors. Canadian Guidelines on Sexually Transmitted Infections. 2010 ed. Ottawa: Public Health Agency of Canada; 2010.

(6) Kropp RY, Wong T, Canadian LGV Working Group. Emergence of lymphogranuloma venereum in Canada. CMAJ 2005 Jun 21; 172(13):1674-1676.

(7) Bremer V, Meyer T, Marcus U, Hamouda O. Lymphogranuloma venereum emerging in men who have sex with men in Germany. Euro Surveill 2006 Sep; 11(9):152-154.

(8) Koedijk FD, de Boer IM, de Vries HJ, Thiesbrummel HF, van der Sande MA. An ongoing outbreak of lymphogranuloma venereum in the Netherlands, 2006-2007. Euro Surveill 2007 Apr 19; 12(4):E070419.2.

(9) Jebbari H, Alexander S, Ward H, Evans B, Solomou M, Thornton A, et al. Update on lymphogranuloma venereum in the United Kingdom. Sex Transm Infect 2007 Jul; 83(4):324-326.

(10) HIV, STD and Hepatitis Prevention Branch, Public Health Services, Health and Human Services Agency, San Diego County. Lymphogranuloma venereum (LGV) update. 2005.

(11) Savage EJ, van de Laar MJ, Gallay A, van der Sande M, Hamouda O, Sasse A, et al. Lymphogranuloma venereum in Europe, 2003-2008. Euro Surveill 2009 Dec 3; 14(48):19428.

(12) Public Health Agency of Canada. Report on sexually transmitted infections in Canada: 2012. Centre for Communicable Diseases and Infection Control, Infectious Disease Prevention and Control Branch, Public Health Agency of Canada; 2015.

(13) Public Health Agency of Canada. A supplementary statement concerning the laboratory diagnosis of LGV. Canadian guidelines on sexually transmitted tnfections. http://www.phac-aspc.gc.ca/std-mts/sti-its/cgsti-Idcits/supp-lgv-eng.php (Disponible en français: http://www.phac-aspc.gc.ca/std-mts/sti-its/cgsti-ldcits/supp-lgv-fra.php).

(14) Public Health Agency of Canada. Case definitions for communicable diseases under national surveillance Can Comm Dis Report. 2009; 35;S-2.

(15) McKay A, Barrett M. Rising reported rates of chlamydia among young women in Canada: What do they tell us about trends in the actual prevalence of the infection? The Canadian Journal of Human Sexuality 2008; 17(1-2):61-69.

(16) Rekart ML, Brunham RC. Epidemiology of chlamydial infection: Are we losing ground? Sex Transm Infect 2008 Apr; 84(2):87-91.

(17) Tuite AR, Jayaraman GC, Allen VG, Fisman DN. Estimation of the burden of disease and costs of genital Chlamydia trachomatis infection in Canada. Sex Transm Dis 2012 Apr; 39(4):260-267.

(18) Lee V, Tobin JM, Foley E. Relationship of cervical ectopy to chlamydia infection in young women. J Fam Plann Reprod Health Care 2006 Apr; 32(2):104-106.

(19) Junior JE, Giraldo PC, Goncalves AK, do Amaral RL, Linhares IM. Uterine cervical ectopy during reproductive age: Cytological and microbiological findings. Diagn Cytopathol 2014 May; 42(5):401-404. 
(20) Riera-Montes M, Velicko I. The chlamydia surveillance system in Sweden delivers relevant and accurate data: Results from the system evaluation, 1997-2008. Euro Surveill 2011 Jul 7; 16(27):19907.

(21) Machalek K, Hanley B, Kajiwara J, Pasquali P, Stannard C. Chlamydia screening practices among physicians and community nurses in Yukon, Canada. International journal of circumpolar health 2013; 72(22447).

(22) Linedegger M, Salway Hottes T, Gilbert M, Lester R, Imperial M, Hoang L. Lymphogranuloma venereum in British Columbia: 2004 to 2011. Epidemiology and Surveillance, Clinical Prevention Services 2012.

(23) Public Health Agency of Canada. Canadian guidelines for sexual health education. Ottawa: Public Health Agency of Canada; 2008. 